\title{
Proposal for a readout driver card for the ATLAS Insertable B-Layer
}

Davide Falchieri ${ }^{1}$, Graziano Bruni², Marco Bruschi², Ignazio D’Antone ${ }^{2}$, Jens Dopke ${ }^{3}$, Tobias Flick ${ }^{3}$, Alessandro Gabrielli ${ }^{1}$, Joern Grosse-Knetter ${ }^{4}$, John Joseph ${ }^{5}$, Nina Krieger ${ }^{4}$, Andreas Kugel6, Paolo Morettini', Alessandro Polini², Matteo Rizzi ${ }^{2}$, Nicolai Christian Schroer ${ }^{6}$, Riccardo Travaglini ${ }^{2}$, Samuele Zannoli ${ }^{1}$, Antonio Zoccoli ${ }^{1}$

\section{University of Bologna \& INFN Bologna, ${ }^{2}$ INFN Bologna, ${ }^{2}$ Fachbereich C Physik, Bergische Universitaet Wuppertal,}

INFN

${ }^{4}$ II. Physikalisches Institut, Universitaet Goettingen, ${ }^{5} \mathrm{LNBL}$, Berkeley, ${ }^{6}$ ZITI, LS Informatik V, Heidelberg University, Mannheim, ${ }^{2}$ INFN Genova

\section{Introduction}

An additional inner layer for the existing ATLAS Pixel Detector, called Insertable B-Layer (IBL), is under design and it will be installed by Phase 1. New front-end readout ASICs fabrication (FE-I4) will replace the previous chips in this layer. The new system features higher readout speed $160 \mathrm{Mb} / \mathrm{s}$ per ASIC - and simplified control. The current data acquisition chains are composed of front-end detectors, readout chips, Back-OfCrate (BOCs) cards and ReadOut Driver cards (RODs). The poster presents a proposal for the new ROD board, which implements modern FPGAs and high-speed links with the detector and with the ATLAS TDAQ system.

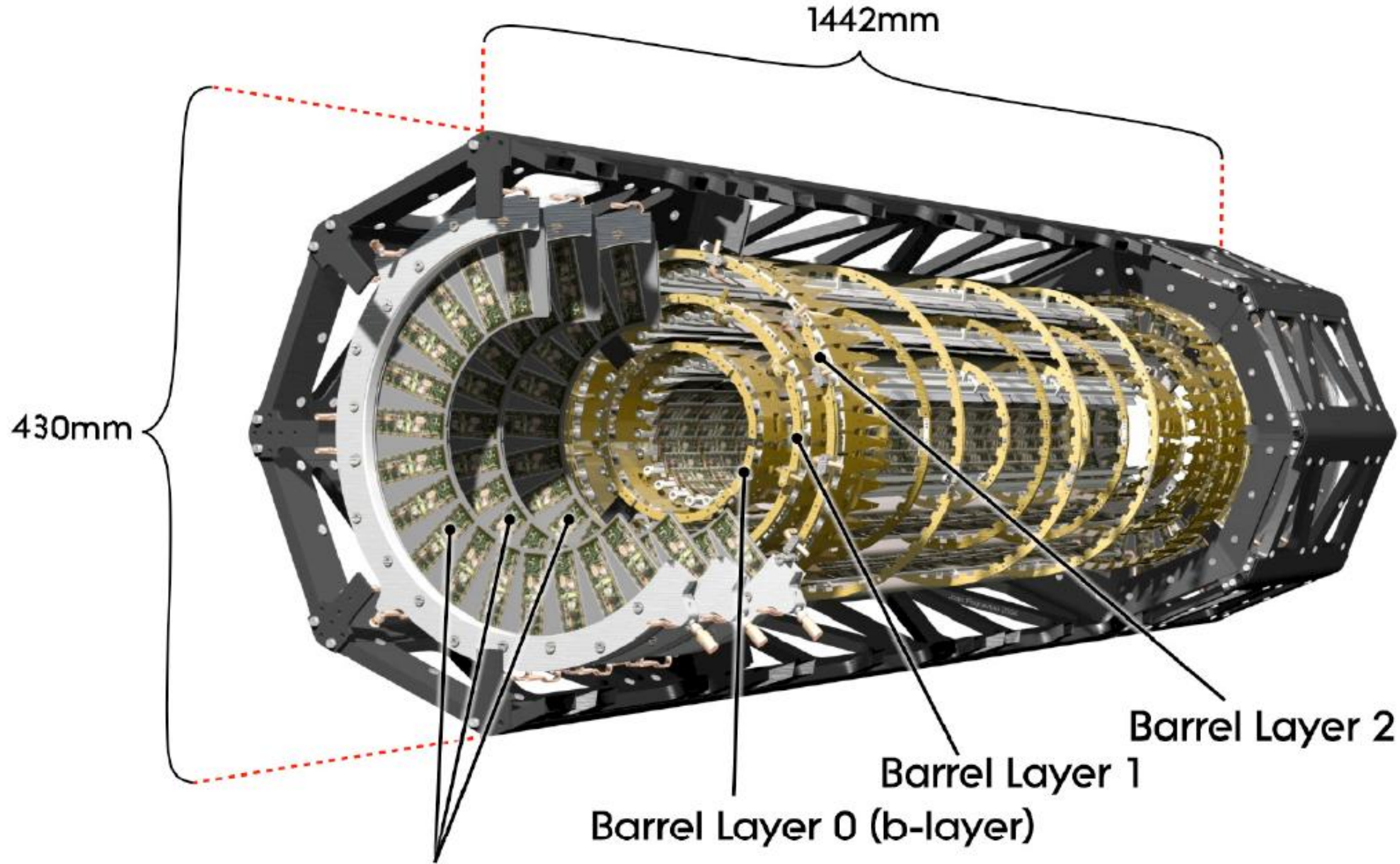

End-cap disk layers

IBL front-end electronics

The Pixel Detector readout architecture was designed to be fully efficient at the "nominal" LHC peak luminosity of $10^{34} \mathrm{~cm}^{-2} \mathrm{~s}^{-1}$ and for a LVL1 trigger rate of 100 $\mathrm{kHz}$. Two bottlenecks arise in the architecture at luminosities greater than nominal, one in the FE-I3 (front-end chip) and one in the link between the MCC (Module Controller Chip) and the off-detector electronics:

- the double-column bus in the FE-I3 (sensitive to the occupancy),

- the link from MCC to the off-detector (sensitive to the product of the hit occupancy and LVL1 rate).

These bottlenecks can give rise to readout inefficiencies that would impact btagging efficiency in the absence of the IBL.

The FE-14 front-end chip has a completely new internal architecture that fulfils the arger request of occupancy and bandwidth.

Main new features:

compression of hit pairs in single hit word

new readout architecture based on $2 \times 2$ pixel regions to improve throughput

-8B/10B output data encoding scheme

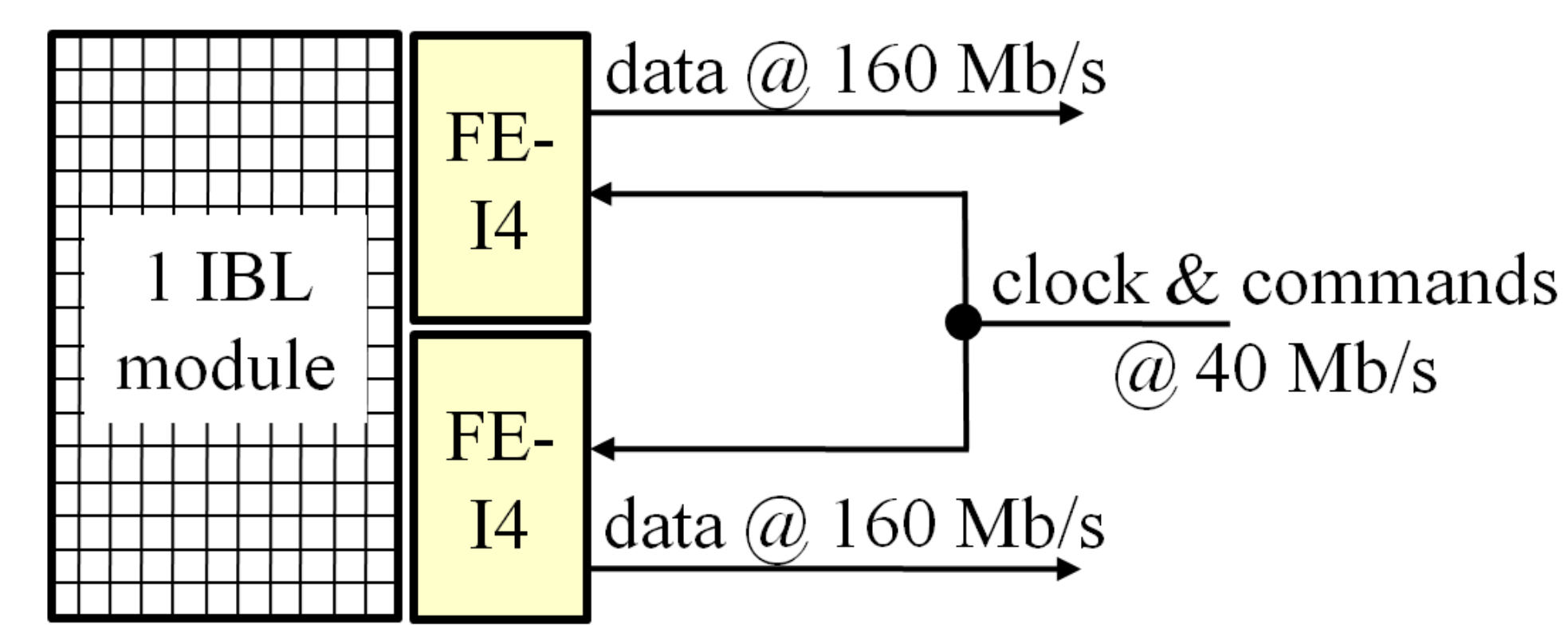

ROD design guidelines

- Hardware compatibility with the current BOC board, an ad-hoc firmware can be developed if required.

- Modularity corresponding to thirty-two $160 \mathrm{Mb} / \mathrm{s}$ input channel and four $160 \mathrm{MB} / \mathrm{s}$ S-Link output channels. Possibility to reduce to eight $160 \mathrm{Mb} / \mathrm{s}$ input links and one output S-Link in compatibility

- Full data path implemented in few large FPGAs, to maximize the design flexibility and simplify the inter-communication between the data path components.

- Increase the output bandwidth for calibration histograms. This can be achieved using high speed serial links (Gigabit Ethernet) for sending calibration data to an external PC farm. In fact a solution with $2 \mathrm{~Gb} / \mathrm{s}$ links per ROD could make it convenient to extract raw histograms immediately and process them on commercial processors, in this way eliminating the need for DSPs on the ROD.

- Use of an embedded PowerPC core (inside a Virtex5 FPGA) performing system control and non real-time functions. In particular it allows to run iterative code executing on-board scans without host intervention. Using an embedded processor should allow to decrease debug times since it allows to use a common simulation environment for both processor and FPGA logic behavior.
ATLAS Pixel Detector:

3 barrels +3 forward/backward disks

1744 modules with 16 FE-I3 chips each

lions readout channels

Petector system is the innermost element of the Inner Detector. It provides pattern recognition capability in order to luminosity of LHC $\left(10^{34} \mathrm{~cm}^{-2} \mathrm{~s}^{-1}\right)$
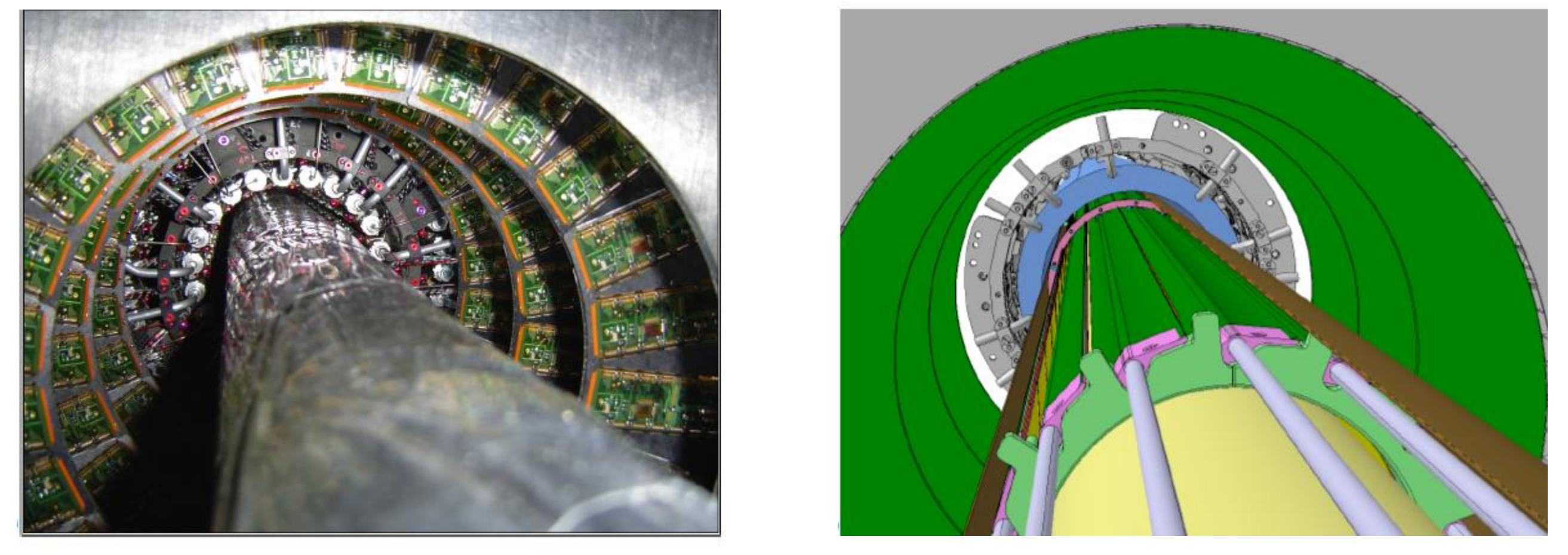

ATLAS IBL (Insertable B-Layer):

- 224 modules with 2 FE-I4 chips each in 14 staves at $3.3 \mathrm{~cm}$ radius

- $\sim 12$ millions readout channels

IBL is a fourth layer added to the present Pixel Detector between a new beam pipe and the current B-layer. IBL will allow to keep robust tracking despite effects arising from luminosity increase, hardware lifetime and radiation effects. IBL will also provide improved precision for vertexing and b-tagging to the existing detector

Proposal of a new off-detector readout

When specifying the design of the DAQ chain for the IBL, the first open question was whether the existing ROD was sufficient or if a new one was needed. The existing ROD firmware could be modified to operate with the IBL module data format; however, the hardware of the board is designed to operate with a maximum of eight $160 \mathrm{Mb} / \mathrm{s}$ input links (from the modules) to one output S-Link, while, in order to respect the IBL natural modularity, thirty-two $160 \mathrm{Mb} / \mathrm{s}$ links to four S-Links have to be handled. This consideration, together with the $4 \mathrm{MB} / \mathrm{s}$ bandwidth limitation on the VME bus, and the obsolescence of the components, led to the decision to design a new BOC-ROD pair.

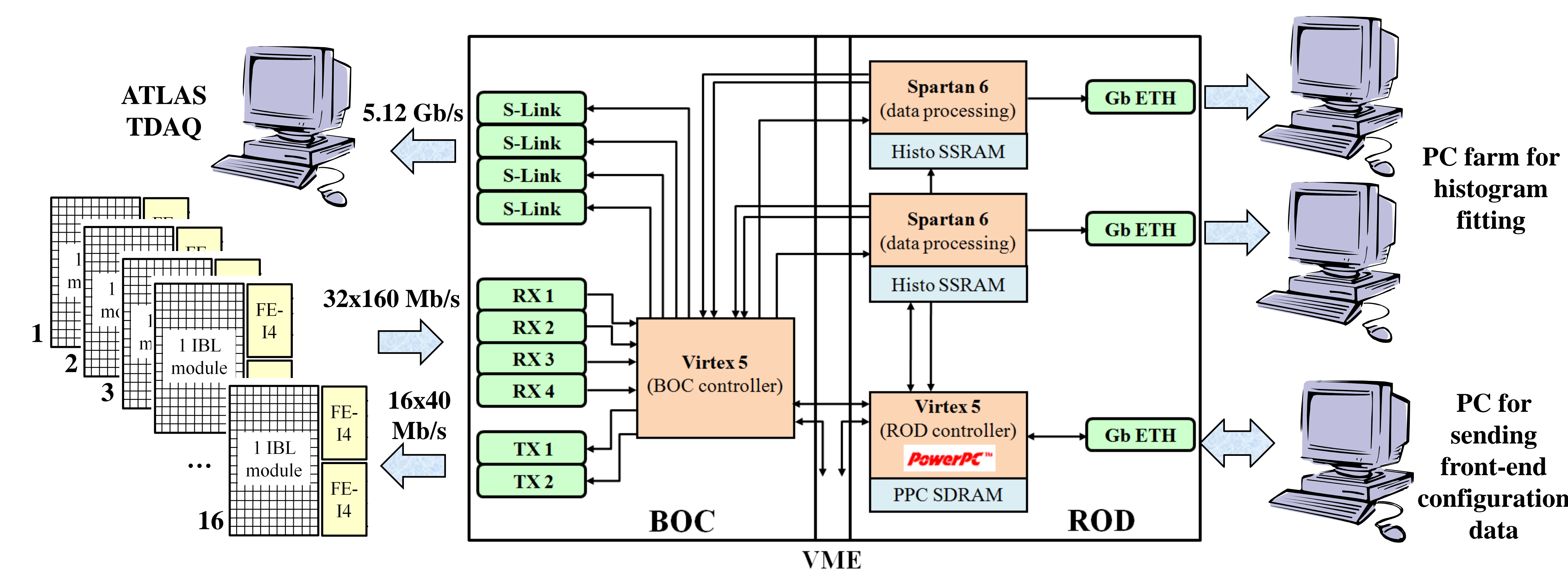

Histogramming block

As for the Pixel Detector, the calibration of the IBL detector is performed by repeating relatively short (100-1000) series of events, recorded while injecting known charge into each pixel, and with different settings of the front-end parameters (e.g. different thresholds or different pre-amplifier feedback currents). This sequence, called calibration scan, generates a very large number of events, which have to be analyzed in order to extract the histogram showing how many times each pixel has been fired for a given setting. For this reason, in calibration mode, the data stream coming from the sensor is not sent over the $\mathrm{S}$ Link, but pre-processed in the ROD, where the relevant histograms are produced. At the end of the scan, the histograms are transferred to an external farm via Gb Ethernet for fitting and archiving.

A novel approach to carry out histograms and analysis on-ROD is proposed. The new ROD will execute only the calibration loops to accumulate the per-pixel occupancies, sums of time-over-threshold (ToT) and sums of ToT $^{2}$ parameters. Then histograms are created and saved on-the-fly on RAMs and eventually transferred via Gb Ethernet to an off-line high-performance computer.
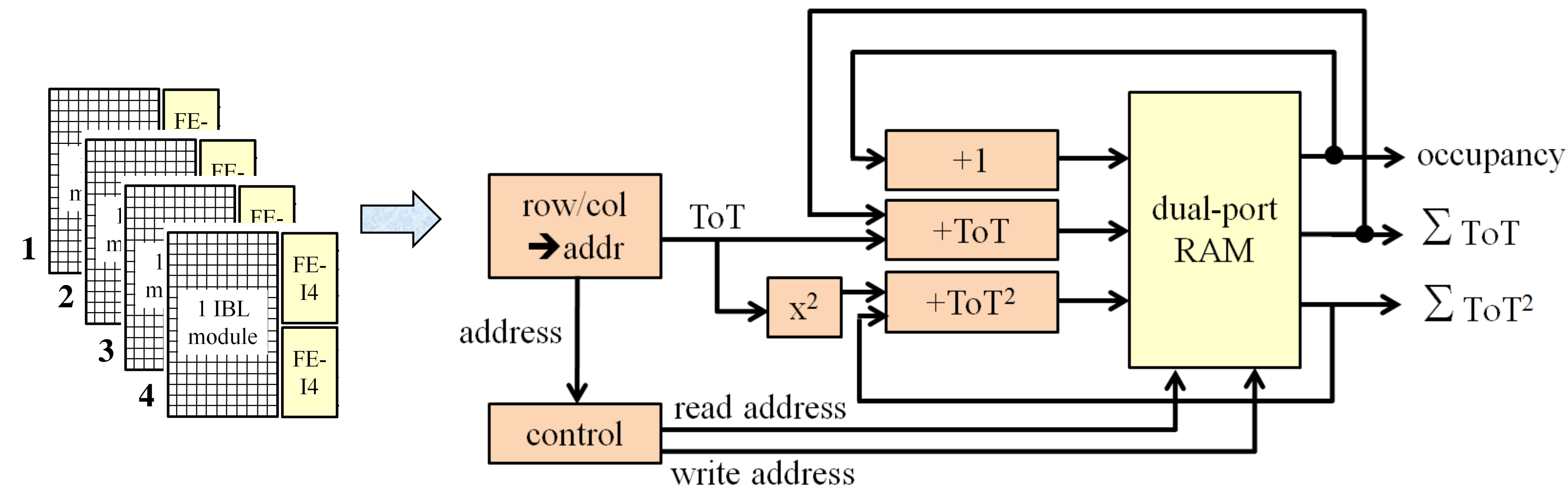

Conclusion

The architecture of the new BOC-ROD boards for the readout of IBL is presented. The boards are currently under design and a firmware test-bed based on Avnet evaluation boards is planned. 